\title{
Esophageal Cancer in Brunei Darussalam over a three Decade Period: an Epidemiologic Study of Trends and Differences between Genders and Racial Groups
}

\author{
Vui Heng Chong1*, Pemasari Upali Telisinghe², Chee Fui Chong ${ }^{3}$
}

\begin{abstract}
Background: Carcinoma of the esophagus is associated with significant morbidity and mortality. The most common subtype is squamous cell carcinoma (SCC). In the past three decades, the incidence of SCC has been reported to be decreasing whereas esophageal adenocarcinoma $(\mathrm{AC})$ is increasing. This study assessed the trend of esophageal cancer in Brunei Darussalam over a three decades period. Materials and Methods: The National Cancer registry was searched for esophageal cancers from 1986 to 2012. Data on age, gender, racial groups (Malays, Chinese, Indigenous and foreign nationals) and histology type were collected. The rate (ASR) and Age Specific Incidence rate (ASIR) were calculated. Results: The predominant tumor type was SCC which accounted for 89\% of all esophageal cancer. The gender ratio was 2.25: 1 (male: female) and the mean age at diagnosis was $66.9 \pm 12.9$ years, significantly younger for esophageal $\mathrm{AC}(57.2 \pm 16.0)$ compared to $\operatorname{SCC}(68.1 \pm 12.0, \mathrm{p}<0.05)$, and among the foreign nationals $(\mathrm{p}<0.05$ for trend). The proportions of SCC among all esophageal cancers in the various racial groups were: Malays $(\mathbf{8 7 . 8 \%})$, Chinese $(\mathbf{1 0 0 \%})$, Indigenous $(\mathbf{1 0 0 \%})$ and foreign nationals (20\%). None of the Chinese and Indigenous groups were diagnosed with esophageal AC. The overall ASR for esophageal cancer was 2.1/100,000; 2.0/100,000 for SCC with a declining trend and $0.17 / 100,000$ for esophageal $\mathrm{AC}$, without any trend observed. Among the two major racial groups; the Chinese has higher ASR (3.42/100,000) compared to the Malays (ASR 0.95/100,000). Conclusions: SCC is the predominant tumor type of esophageal cancer in Brunei Darussalam and more common among the Chinese. There was a declining trend in the incidence of SCC but not for esophageal AC.
\end{abstract}

Keywords: Esophageal cancer - squamous cell carcinoma - adenocarcinoma - presentations - incidence

Asian Pac J Cancer Prev, 16 (9), 4123-4126

\section{Introduction}

Cancer of the esophagus is uncommon compared to cancers of other part of the gastrointestinal tract, and consists primarily of two main histological types; squamous cell carcinoma (SCC) and adenocarcinoma (AC). The SCC type is usually located in the proximal two third whereas the $\mathrm{AC}$ is often found near the gastroesophageal junction. Smoking and alcohol are important risk factors for SCC whereas Barrett's esophagus secondary to chronic acid injuries is an important risk factor for AC (Falk, 2009). Gender, ethnicity, age, family history of similar cancers, oesophageal corrosive injuries and achalasia are other important risk factor (Falk, 2009).

The overall prognosis of esophageal carcinoma remains poor as most are diagnosed at the advanced stages (Falk 2009) resulting in significant mortality. A recent study from China showed that the mortality trend had been increasing (Tang et al., 2014). In 2012, an estimated 455,784 cases of esophageal cancers were recorded with an Age Standardized Rate (ASR) was
$5.9 / 100,000$, with $81 \%(369,640)$ occurring in the less developed regions (IARC, 2012). Of these, there were 400,169 deaths, giving a mortality rate of over $87 \%$ (ASR of 5.0/100,000 for mortality). Countries with high ASRs include; Malawi 24.2/100,000, Kenya 17.6/100,000 and Uganda 17.1/100,000 in Eastern Africa, Turkmenistan $(19.7 / 100,000$, Tajikistan $(14.7 / 100,000)$ and Bangladesh $(12.7 / 100,000)$ in South-Central Asia, and Mongolia $(17.6 / 100,000)$ and China $(12.5 / 100,000)$ in Eastern Asia (IARC 2012), all underdeveloped or developing nations. However, in large countries such as China, the rates vary between regions and higher in the rural compared to the urban areas (Feng et al., 2014). In 2012, the rates in South-East Asia varied from 0.6/100,000 in Lao DPR to 7.6/100,000 in Myanmar.

Recent reports have indicated that the incidence of $\mathrm{AC}$ has increased during the last two decades whereas the incidence of SCC is decreasing (Vega and Jamal 2000; Fernandez et al., 2006; He et al., 2008; Trivers et al., 2008; Cook et al., 2009). This study assesses the trend of esophageal cancers in Brunei Darussalam a developing 
Vui Heng Chong et al

South-East Asian nation with a predominant Malay population over a period of three decades.

\section{Materials and Methods}

Patients with histology proven esophageal carcinoma (SCC and AC) diagnosed between 1986 and 2012 were identified from the cancer registry maintained by the Department of Pathology, RIPAS Hospital, the only state laboratory that handled all tissue specimens. Therefore, this registry captures all the histology proven cancers for the whole country. Demographic and tumor data was extracted. The data between this time periods was used to assess the trend of esophageal carcinoma. The data was presented as the Age Standardized Rate (ASR) and Age Specific Incidence Rate (ASIR) based on the projected population for Brunei (Population census department, Ministry of Finance) and the world population (WHO data). The age specific incidence rates were calculated based on the population of 1999. Data on age were not available for 1984 and 1985 and were excluded from analyses of the ASR and age specific incidence rates. The rates for genders, the two major ethnic groups (Malays and Chinese) and the tumor types (SCC and AC) were calculated.

\section{Statistical analyses}

The data were coded and entered into the Word Excel program and later entered into the SPSS (version 10.0, Chicago, IL, USA) for analysis.

\section{Results}

Over the study period (1986-2012), there were 91 cases of esophageal cancers diagnosed. The overall mean age at diagnosis was $66.9 \pm 12.9$ years. All presented with dysphagia and weight loss of several months. All were either locally advanced or with metastases at diagnosis. The demographic and tumor types are shown in Table 1.

Esophageal AC was diagnosed in the Malays (12.2\%) and the Foreign nationals $(80 \%)$, and none in the Chinese and the Indigenous group (Figure 1). All the esophageal cancers diagnosed in Foreign nationals were all in men.

Among the genders, the proportion of SCC among the Chinese and the Indigenous group were (men $84.9 \%$; women $15.1 \%$ ) and (men $75 \%$; women $25 \%$ ) respectively, and for the Malays; SCC (men 55.8\%; women 44.2\%) and esophageal AC (men 50\%; women 50\%).

The overall ASR for esophageal cancer was $2.1 / 100,000$, with the latest rate of $0.85 / 100,000$ for the 2011-2012 period. The overall ASR for SCC was 2.0/100,000 with a declining trend and for EAC was $0.17 / 100,000$ without any trend observed (Figure 2).

Among the two major racial groups; the Chinese has higher ASR $(3.42 / 100,000)$ compared to the Malays (ASR 0.95/100,000).

The ASIRs were two to three times higher for men compared to women, highest in the 65-69 age groups (Figure 3). Between the two major ethnic groups, the Chinese had higher rates than the Malays, almost reaching four times more common in the highest age groups (70 years or more groups) (Figure 4).

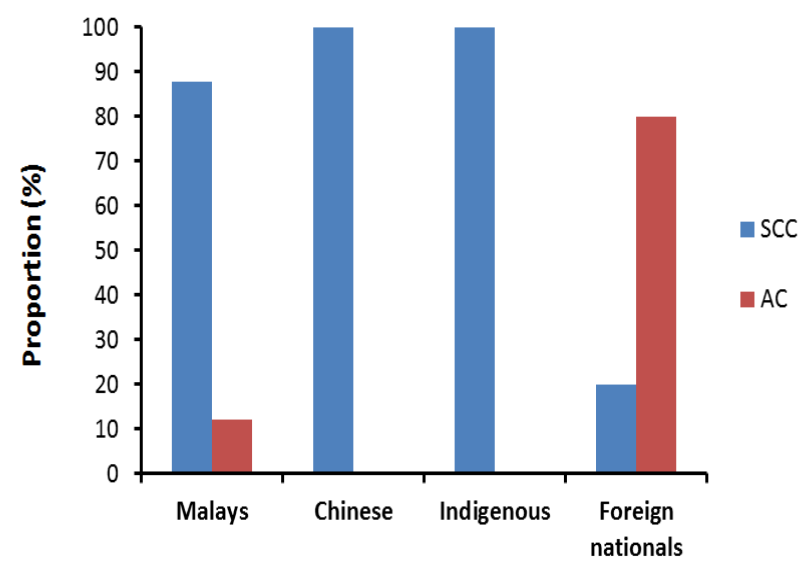

Figure 1. Breakdown in the Proportion of SCC and AC among the Various Groups

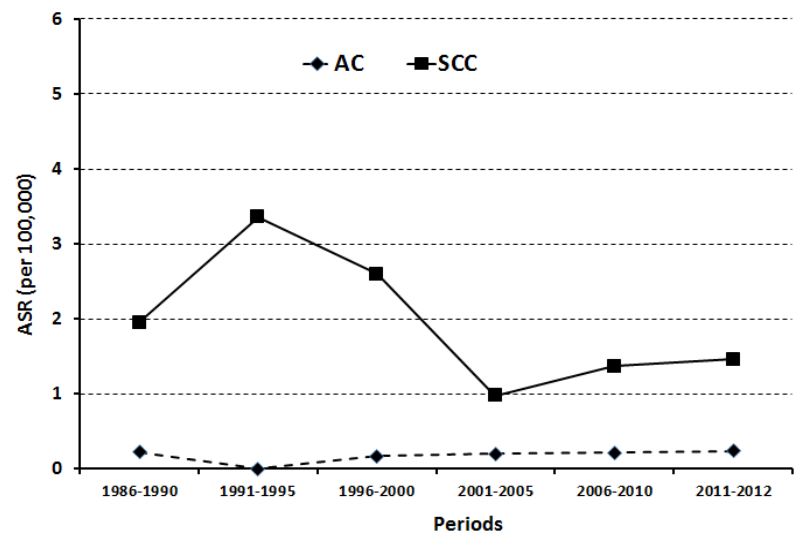

Figure 2. The ASR for the SCC and EAC Over the 27 years in 5-years block

Table 1. Demographics and the Tumor Types

\begin{tabular}{lrcc}
\hline Characteristics & $\mathrm{n}(\%)$ & Mean age (years) & $\mathrm{p}$ value \\
\hline Racial groups & & & $<0.05$ \\
$\quad$ Malays & $47(53.8)$ & $68.3 \pm 11.6$ & for trend \\
Chinese & $33(36.3)$ & $66.9 \pm 15.4$ & \\
$\quad$ Indigenous & $4(4.4)$ & $64.5 \pm 2.6$ & \\
$\quad$ Others & $5(5.5)$ & $54.6 \pm 7.8$ & $>0.05$ \\
Gender & $63(69.2)$ & $66.3 \pm 13.1$ & \\
$\quad$ Men & $26(30.8)$ & $68.2 \pm 12.7$ & $<0.05$ \\
$\quad$ Women & $77(89)$ & $68.1 \pm 12.0$ & $57.2 \pm 16.0$ \\
Tumor types & $10(11)$ & & \\
$\quad$ Squamous cell carcinoma (SCC) & & \\
Adenocarcinoma (AC) &
\end{tabular}




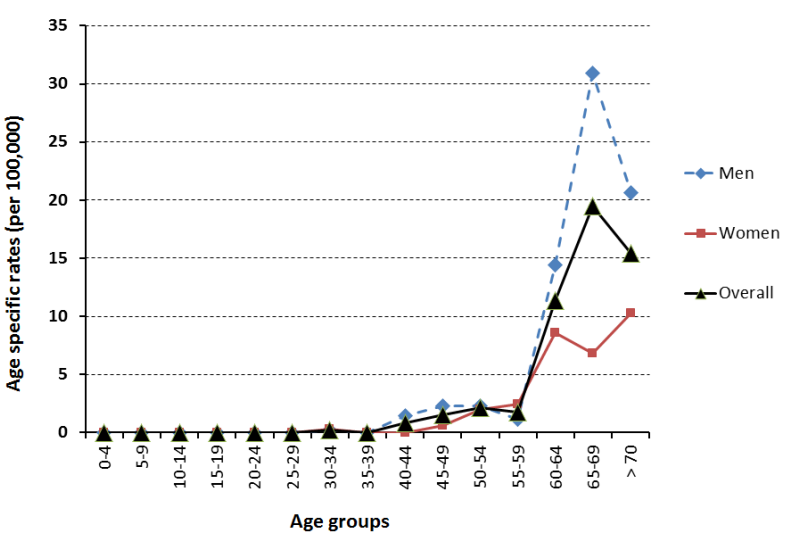

Figure 3. The age Specific Incidence Rates (overall; Black with triangular Marker, and Genders; Men with Broken Blue Line and Women With Red Line)

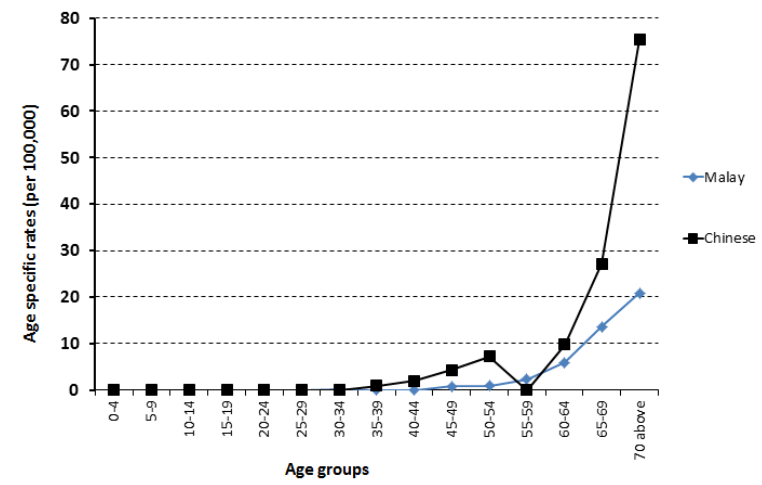

Figure 4. Age Specific Incidence Rates between the Two Major Ethnic Groups (Malays; blue line and Chinese; Black Line)

\section{Discussion}

Similar to other countries (IARC 2012), esophageal cancers are uncommon in our Brunei Darussalam compared to other cancers (Mohamad et al., 2014).Among cancers of the gastrointestinal tract, esophageal cancers ranked as one of the least common compared to colorectal, stomach, liver, biliary and pancreas in decreasing order (IARC, 2012, Mohamad et al., 2014). Based on the present study, our rates were low compared to rates reported in the literature (Fernandez et al., 2006; He et al., 2008; Trivers et al., 2008; Cook et al., 2009; IARC 2012). Compared to the other South-East Asian nations, our rate (2011-2012, ASR 0.85/100,000), was lower than the rates reported for the South-East Asian region (ASR 2.0/100,000), ranging from a low of $0.6 / 100,000$ in Lao DPR to a high of 7.1/100,000 in Myanmar (IARC 2012). Overall, Brunei has the second lowest rate for esophageal cancers in the South-East Asia region. Generally the incidence are high in the less developed countries (IARC 2012), also reflected in the higher rates in the rural compared to urban areas (Feng et al., 2014).

Over the last three decades, the incidence of SCC has decreased while the incidence of esophageal AC has increased especially in the more developed nations (Fernandes et al., 2006, Trivers et al., 2009). However, the increase for esophageal AC is small. In agreement with what have been reported (Fernandes et al., 2006;
He et al., 2008; Trivers et al., 2009; Cook et al., 2009; Wang et al., 2013, Feng et al., 2014), there is a decline in the overall incidence rate for esophageal cancers in our local setting, especially for SCC. This is likely to be related to improvement in the standard of living. In a study from Singapore, the reported ASRs for SCC had shown a significant decline from 8.31 to 3.85 per 100,000 men $(\mathrm{p}=0.017)$ and from 3.43 to 0.81 per 100,000 women $(\mathrm{p}=0.027)$ (Fernandes et al., 2006). The study also reported increasing trend for esophageal $\mathrm{AC}$ from 0 to 0.54 per 100,000 men and from 0.03 to 0.13 per 100,000 women. No such trend was observed for esophageal AC in our local setting. In fact the overall number of cases has remained low in our local setting. For esophageal AC, the highest proportion was among the Foreign nationals (80\%) and none were diagnosed among the Chinese and the Indigenous groups.

The increasing trend of $\mathrm{AC}$ reported in the literature had been attributed to the increase in the incidence of Barrett's esophagus (Falk, 2009; Hongo et al., 2009; Horváthet al., 2009). In our previous study, we showed that endoscopic Barrett's esophagus was rare (Chong, 2008), but since we have noticed an increase in the incidence, but only a small increase. To date, none of our cases with esophageal AC had been associated Barrett's esophagus. Recent evidence has shown the risk of progression from Barrett's esophagus to AC has been estimated to be 0.4 to $0.5 \%$ per year, much lower than the previously estimated 1 to $2 \%$ per year. Therefore, other factors are important. Obesity has been linked with the increasing incidence of esophageal AC (Murray et al., 2009). Other factors such as diet (diet rich in nitrosamine, meat and low in fruits and vegetables) (Stefani et al., 2014), tea consumption, tobacco, alcohol and lacks of certain micronutrients are important (Falk et al., 2009). Of this, tobacco use and consumption of salted food are common in our local setting.

Similar to what have been reported, we also showed that esophageal carcinoma is a male predominant disease (Fernandez et al., 2006; He et al., 2008; Trivers et al., 2008; Cook et al., 2009; IARC 2012). Almost two third of our patients were men. This is not surprising as smoking, a risk factor for SCC is common among men. The estimated incidence of tobacco use (active smokers and ex-smokers) in our local setting was $44.6 \%$ for adult male and $8.6 \%$ for adult female based on the National Nutrition Status Survey-1997. Use of tobacco was lowest among the Chinese. Similarly, alcohol consumption is also more common among men.

Another finding of our study which will be important for national health policy planning is the ethnic differences. The racial breakdown for esophageal cancers was $52.5 \%$ for Malays, $35.0 \%$ Chinese and $12.5 \%$ for Indigenous group compared to the population breakdown; (Malays 70\%), Chinese (12\%) and the indigenous group $(\leq 5 \%)$. This suggests that both the non-Malays have other risk factors for developing esophageal carcinoma. Apart from shared environmental factors, genetic and culturally associated environmental factors are important. Our previous studies have also shown that our population Chinese had higher incidence of gastric and colorectal, 
suggesting inherent risk associated with Chinese ethnicity (Chong et al., 2009; Chong et al., 2014). Differences in the incidence of esophageal cancers have been reported between the ethnic groups with countries (Cook et al., 2009; Fitzgerald et al., 2009; Coupland et al., 2012) Studies from the United States have also consistently reported ethnic backgrounds to be an important risk factor with black American at higher risk for SCC and white American for esophageal AC (Cook et al., 2009; Fitzgerald et al., 2009). A study from the United Kingdom looking at esophageal cancers diagnosed between 2001 and 2007 reported variation in cancer incidence between the different ethnic groups (Coupland et al., 2012). Compared to White men, the incidence rate ratios (IRR) for the other ethnic groups were lower (Indian IRR 0.42, Pakistani IRR 0.17, Bangladeshi IRR 0.39, Black Caribbean IRR 0.58, Black African IRR 0.39, and Chinese IRR 0.36). Compared with White women, the incidence were higher among Bangladeshi women (IRR 2.02) and lower among African women (IRR 0.86) Indian (IRR 0.68), Pakistani (IRR 0.26), Black Caribbean (IRR 0.56), and Chinese (IRR 0.42). Therefore, the ethnic background of the population is important.

Based on the findings of our study, an important step is to increase public's awareness through various programs. Almost all of our esophageal cancer patients presented late with the locally advanced disease making curative resections not feasible. Screening, which has been shown to be effective in high incidence country (Wang et al., 2013) will not be required or cost effective in our setting. The Brunei Government has already implemented the Tobacco Order 2005 as part of the WHO Framework Convention on Tobacco Control in 2005 with tight regulation of tobacco sale and premises designated as nonsmoking areas. This may eventually lead to stricter control and even complete ban of tobacco sales in the country. This important measure will not only reduce further the number of patients with esophageal cancers in the future, but also other tobacco related cancers and non-communicable diseases such as stroke, ischemic heart disease and chronic obstructive airway disease.

In conclusion, there is a decline in the overall ASR for esophageal cancer, especially SCC. The Chinese population has higher risk compared to the other ethnic groups. Most were still diagnosed at advanced stages. More needs to be done to address the issue of late presentations so that diagnoses can be made early to improve the outcomes of oesophageal carcinoma in Brunei Darussalam.

\section{References}

Chong VH (2008). Findings among patients referred for endoscopy in Brunei Darussalam with special reference to dyspepsia. Brunei Darussalam J Health, 3, 24-31.

Chong VH, Telisinghe PU, Abdullah MS, Chong CF (2014). Gastric cancer in Brunei Darussalam: epidemiological trend over a 27 year period (1986-2012). Asian Pac J Cancer Prev. 15, 7281-5.

Chong VH, Telisinghe PU, Abdullah MS, Jalihal A (2009). Colorectal cancer: incidence and trend in Brunei Darussalam. Singapore Med J, 50, 1085-9.
Cook MB, Chow WH, Devesa SS (2009). Oesophageal cancer incidence in the United States by race, sex, and histologic type, 1977-2005. Br J Cancer, 101, 855-9.

Coupland VH, Lagergren J, Konfortion J, et al (2012). Ethnicity in relation to incidence of oesophageal and gastric cancer in England. Br J Cancer, 107, 1908-14.

De Stefani E, Deneo-Pellegrini H, Ronco AL, et al (2014). Diet patterns and risk of squamous cell oesophageal carcinoma: a case-control study in Uruguay. Asian Pac J Cancer Prev, 15, 2765-9

Falk GW (2009). Risk factors for esophageal cancer development. Surg Oncol Clin N Am, 18, 469-85.

Feng XS, Yang YT, Gao SG, et al (2014). Prevalence and age, gender and geographical area distribution of esophageal squamous cell carcinomas in North China from 1985 to 2006. Asian Pac J Cancer Prev, 15, 1981-7.

Fernandes ML, Seow A, Chan YH, Ho KY (2006). Opposing trends in incidence of esophageal squamous cell carcinoma and adenocarcinoma in a multi-ethnic Asian country. Am J Gastroenterol, 101, 1430-6.

Fitzgerald TL, Bradley CJ, Dahman B, Zervos EE (2009). Gastrointestinal malignancies: when does race matter? $J$ Am Coll Surg. 209, 645-52.

He YT, Hou J, Chen ZF, et al. Trends in incidence of esophageal and gastric cardia cancer in high-risk areas in China. Eur $J$ Cancer Prev, 17, 71-6.

Hongo M, Nagasaki Y, Shoji T. Epidemiology of esophageal cancer: Orient to Occident. Effects of chronology, geography and ethnicity. J Gastroenterol Hepatol, 24, 729-35.

International Agency for Research on Cancer. Globocan 2012: Estimated cancer incidence, mortality and Prevalence Worldwide 2012. Available from http://globocan.iarc.fr/ Pages/fact_sheets_cancer.aspx (Accessed 15th August 2014).

Mohammad IA, Bujang MR, Telisinghe PU, Abdullah MS, Chong CF, Chong VH (2014). Cancers of the young population in Brunei Darussalam. Asian Pac J Cancer Prev, 15, 6357-62.

Murray L, Romero Y (2009). Role of obesity in Barrett's esophagus and cancer. Surg Oncol Clin N Am, 18, 439-52.

Tang WR, Fang JY, Wu KS, Shi XJ, Luo JY, Lin K (2014). Epidemiological characteristics and prediction of esophageal cancer mortality in China from 1991 to 2012. Asian Pac J Cancer Prev, 15, 6929-34.

Trivers KF, Sabatino SA, Stewart SL (2008). Trends in esophageal cancer incidence by histology, United States, 1998-2003. Int J Cancer, 123, 1422-8.

Vega KJ, Jamal MM (2000). Changing pattern of esophageal cancer incidence in New Mexico. Am J Gastroenterol, 95, 2352-6.

Wang X, Fan JC, Wang AR, et al (2013). Epidemiology of esophageal cancer in Yanting - regional report of a national screening programme in China. Asian Pac J Cancer Prev, 14, 2429-32. 